\title{
Quantifying the Burden of Shoulder and Hip Pain In Water Polo Players Across Different Playing Levels
}

\author{
Michael Girdwood, BHSc, M. Physio Prac ${ }^{1}$, Marilyn Webster, Dip. ASc, BEd, M. Sports Physio, DClin Physio² \\ ${ }^{1}$ La Trobe Sport \& Exercise Medicine Research Centre, La Trobe University, ${ }^{2}$ The University of Melbourne \\ Keywords: water polo, shoulder, overuse, hip, groin \\ https://doi.org/10.26603/001c.18801
}

International Journal of Sports Physical Therapy

Vol. 16, Issue 1, 2021

\section{Background}

Water polo is characterised by unique skills and movements with high demands of both the upper and lower limb. There is growing recognition of the problems of shoulder and hip/groin symptoms in this population.

\section{Purpose}

To quantify the prevalence of shoulder and hip/groin pain in water polo players, and to describe how performance and participation were impacted. Secondary aims investigated whether demographic or training variables were associated with levels of symptoms.

\section{Design}

In-season, cross-sectional questionnaire study

\section{Methods}

An online questionnaire was distributed to all adult levels of the Australian water polo community. Participants were asked about demographic and playing history, and then specific injury history at both the shoulder and hip/groin. Each respondent completed an Oslo Sports Trauma Research Centre (OSTRC) overuse injury questionnaire for the shoulder and hip/groin. Point prevalence and past history were calculated, as well as a morbidity score from OSTRC responses. Risk ratios were used to determine differences between playing levels and sex.

\section{Results}

One hundred, fifty-three respondents completed the questionnaire ( $57 \%$ female). High rates of shoulder pain were reported (38.1\% current, $81.2 \%$ past history), as well as hip/ groin pain (33.1\% current, $60.4 \%$ past history). Current shoulder pain was a risk factor for hip/groin pain (RR 1.99 (95\%CI 1.27-3.12), and hip/groin pain was a risk factor for shoulder pain (RR 1.70 (95\%CI 1.23-2.35). Elite-level athletes had higher prevalence (RR 1.87 [95\%CI 1.01-3.46]) and past history of hip/groin pain (RR 1.76 [95\%CI 1.32-2.36]).

\section{Conclusions}

This is the first study to quantify high self-reported levels of hip/groin pain in water polo athletes. Such high levels may be explained by high amounts of eggbeater kick, especially during skeletal development in adolescence. Shoulder pain continues to be the most common source of injury burden in water polo. Future research should determine whether any modifiable risk factors exist that may reduce the burden of injury in this population.

\footnotetext{
a Corresponding author:

Michael Girdwood

La Trobe Sport \& Exercise Medicine Research Centre

La Trobe University

Bundoora VIC 3086

AUSTRALIA

m.girdwood@latrobe.edu.au
} 


\section{Level of Evidence}

$2 \mathrm{~b}$

\section{INTRODUCTION}

Water polo combines technical ball skills and powerful throwing, with agile swimming and explosive speed. The sport is made up of a unique set of movements, including the eggbeater kick, which forms the platform for many skills and techniques in water polo. Because of these unique movement patterns, it has an injury profile different from swimming, and one that has not been extensively researched. A high prevalence of shoulder pain is generally accepted amongst players and coaches; however, other injuries including hip and groin issues are under-appreciated.

There is a growing recognition of the impact of hip and groin pain in water polo players, ${ }^{1}$ as well as greater understanding of these conditions in musculoskeletal research in general. ${ }^{2}$ Despite this, no studies have attempted to quantify prevalence or burden of hip/groin pain in water polo players. It is also unclear whether presentations in water polo players are different from athletes of other sports with high rates of hip and groin injuries, such as football or ice hockey. It is theorized that the high amount of time spent eggbeater kicking may be implicated in development of symptoms. ${ }^{3,4}$ The eggbeater kick involves rapid cyclical revolutions of the feet, with the movement creating an inward rotation cycle. ${ }^{5}$ This results in repetitive internal and external rotation at the hip, in combination with hip flexion and abduction. Water polo players spend large amounts of time in games and training performing the eggbeater kick, as it is a crucial determinate of performance, ${ }^{3}$ akin to jumpheight in a volleyball player.

Two systematic reviews have investigated shoulder injuries in water polo players. ${ }^{6,7}$ Shoulder pain is the most common injury afflicting water polo players with a prevalence reported between $24-80 \% .{ }^{6}$ A number of authors have proposed mechanisms behind shoulder pain, including range of motion restrictions, ${ }^{8}$ strength or strength ratio deficits, ${ }^{9}$ and workload issues (specifically shooting loads), ${ }^{10}$ which is in line with other overhead or throwing sports. Unfortunately, most research in this area is of low quality, and limited to small observational studies. Also, many studies have been confined to elite levels or national teams, which may make generalizing findings less reliable to recreational populations. Larger scale studies of water polo players, such as those summarizing injuries at major championships or Olympics do not take into account injuries that do not result in time loss. ${ }^{11}$ This may underestimate the burden, particularly as anecdotally many players report "pushing through" symptoms. Performance impacts of pain also have not been captured, which represents an area from which teams could gain significant benefit. While screening for injury remains a controversial topic in sports medicine, ${ }^{12}$ maximizing player performance remains the cornerstone of the profession. The first step to mitigating injuries is understanding the nature of the burden.

The aims of this study were to quantify the levels of shoulder and hip/groin pain in water polo athletes across different levels of participation, and to describe how perfor- mance and participation were impacted. A secondary aim was to investigate whether demographic or training variables were associated with levels of symptoms.

\section{METHODS}

For this in-season cross sectional study, participants were invited to complete an online questionnaire (Google Forms, Google, USA). The questionnaire was advertised through a variety of channels to ensure a wide and representative range of athletes were captured. All national and state water polo bodies were contacted with promotional material to share via social media, email newsletters, and website advertisements. Two follow up emails were sent to ensure all community members were aware of the study. Word of mouth and local player networks were also utilized. The data collection period was from November 2016- March 2017 , in order to capture as many responders as possible while still being in-season, as different States in Australia have slightly different seasonal periods. In 2016/17 there were 6437 senior $(>18)$ registered members of Water Polo Australia (WPA). Participants were required to be over the age of 18 , and be an active water polo player in Australia defined as having played water polo within the prior three months. Informed consent was gained before participants could complete answers to the questionnaire. The study was approved by The University of Melbourne Human Research Ethics (ID: 1648247).

Responders who consented and were within the inclusion criteria were asked to complete the questionnaire, which took around 5-10 minutes. The form was broken into two sections: demographics and playing history, followed by injury history.

Participants were asked to describe their current (prior three months) and previous highest playing level, from a selection of five categories:

- International/Olympic level

- International league

- Junior international level (such as Junior World Championships)

- Australian National water polo league (national competition in Australia)

- Local/regional level - state or lower-level competitions in Australia

For each of these, examples were provided for each competition to ensure players understood what each level corresponded to. It was possible to select multiple responses for the current playing level question, though only the highest level was used for analysis. In addition to this, responders were asked to answer how many years they had been playing water polo (regardless of level).

Responders were then asked to complete a section on training loads;

- Water polo load: Current average number of water polo sessions completed per week (including training and games) - as a numerical value 
- Current average hours of water polo, both training and games, completed per week - as a selection of six categories for convenience, ranging from 2 hours or less to 15 hours or more

- What other forms of physical activity they engaged in weekly (if any) - descriptive form

- Other exercise load: How many hours they spent completing this other physical activity each week - selection of six categories for convenience, ranging from 0 hours, to 10 or more hours.

Following this, participants were asked specific injury history questions relating to shoulder pain, and also hip/ groin pain. Participants were asked about whether they currently had any shoulder pain relating to water polo in the past week (Yes/No), and whether they had ever had shoulder pain previously in their career related to water polo (Yes/ No). The same questions were repeated, but instead for hip/ groin pain. The terminology 'hip/groin', was deliberately used to ensure all presentations were captured, as specific diagnoses or subgroups were not of interest. This aimed to determine what level dysfunction in the hip/groin region is present in water polo athletes. Participants were dichotomised based on these answers as having current pain, or a past history of pain for both body regions.

Following this, responders completed an Oslo Sports Trauma Research Centre (OSTRC) overuse injury questionnaire for both the shoulder and hip/groin. The OSTRC is a valid and reliable method for capturing overuse injuries, as it does not rely on a time-loss definition of injury. ${ }^{13}$ It instead employs four categorical questions around: (1) difficulty participating, (2) reduced training volumes, (3) performance, and (4) pain. Responses total a maximum of 100 , with 0 indicating the optimal score of no symptoms or affected performance, and 100 being the worst possible score.

Finally, participants were asked to briefly describe whether they were currently seeking help from a medical professional for a water polo related injury, and whether they had ever completed an injury prevention program designed specifically for water polo players.

Responses from the questionnaire were automatically compiled into a spreadsheet by Google Forms. Once the data collection period was complete, the spreadsheet was downloaded and worded responses were coded where required to allow for statistical analysis. Information from the questions relating to playing level were transcoded into three categories for analysis; (1) International level (international competition, international league and junior international, (2) National level (national water polo league); and (3) local level (local/regional competitions). All analysis was completed in SPSS (v24.0.0, IBM, Somers, New York). The alpha level was set at 0.05 for all testing.

A score for injury morbidity was calculated by totalling the response of the four OSTRC questions (separately for both shoulder and hip/groin). Point prevalence and past history were calculated based on dichotomous questions in the injury history section of the questionnaire. These were conducted for each playing level group, and risk ratios (RR) were calculated to compare differences between groups. A substantial injury was defined from Questions $2 \& 3$ of the OSTRC - by selecting moderate or severe loss of perfor-

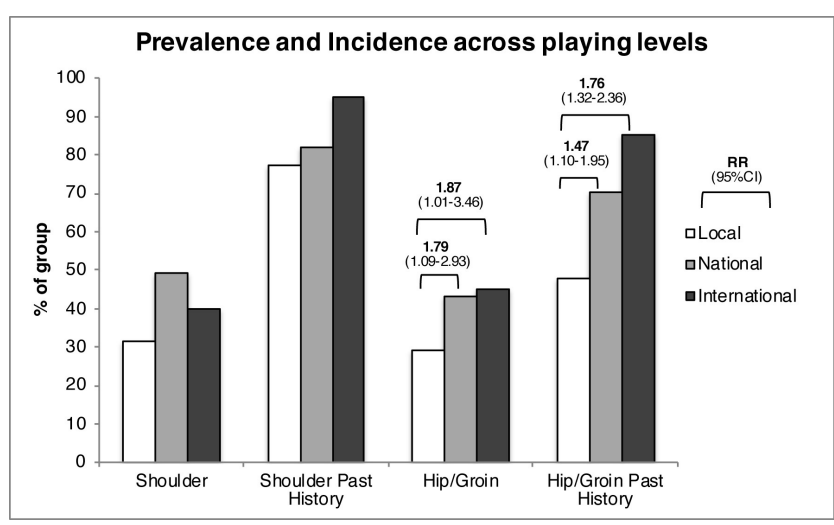

Figure 1: Percentage of study cohort reporting shoulder and hip/groin symptoms

mance or complete inability to participate in sport. ${ }^{13} \mathrm{Re}$ sponders were also classified as having performance impacted, if their response to OSTRC Question 3 (detailing performance) was greater than 0 .

To investigate associations between the continuous variables of age, playing history (years), sessions/week and the prevalence of pain, a point-biserial correlation was conducted. For categorical variables of current and past playing level, water polo load, and other exercise load, a rank-biserial correlation was used. Risk ratios were calculated for shoulder pain as a risk factor for hip/groin pain and vice versa. No analysis was conducted using morbidity scores for the shoulder and hip/groin, as the OSTRC cannot be interpreted as a continuous score.

\section{RESULTS}

One hundred and fifty-three participants completed the questionnaire, with $57.1 \%(\mathrm{n}=88)$ being women. Eightythree local/regional level athletes, 51 national level athletes, and 20 international level athletes completed the questionnaire. Demographic characteristics are presented in Table 1. The median age of responders was 23 (IQR 12), with median years played 10 (IQR 9), and median water polo sessions per week of 4 (IQR 3).

High rates of shoulder and hip/groin pain were reported (Figure 1), with $38.1 \%$ of responders reporting current shoulder pain, and $81.2 \%$ a past history of shoulder pain. Hip/groin pain rates were slightly lower - 33.1\% currently, and $60.4 \%$ reporting a past history. Differences were seen between men and women for hip/groin prevalence and past history (Table 2); however, risk ratios were not significantly different ( $\mathrm{p}=0.33$ and 0.12 respectively). Analysis of differences between prevalence and past history across playing levels is presented in Figure 2. Significant differences in prevalence and past history of hip/groin pain were seen for more elite levels compared to local/regional level athletes.

The median OSTRC for athletes reporting shoulder pain was 28 (IQR 22) and for hip/groin pain was 28 (IQR 22). Twenty-four respondants (15\%) reported a substantial shoulder condition, while 19 (12\%) a substantial hip condition. Thirty-five players (22.7\%) reported concurrent shoulder and hip/groin pain. The presence of shoulder pain was 
Table 1: Demographic characteristics of sample, broken down by playing level. All values are median (IQR) unless denoted so.

\begin{tabular}{|l|l|l|l|l|}
\hline & Local/Regional $\mathbf{n = 8 3}$ & National $\mathbf{n = 5 1}$ & International $\mathbf{n = 2 0}$ & Total $\mathbf{n}=\mathbf{1 5 4}$ \\
\hline \% Female & $52 \%(n=43)$ & $63 \%(n=32)$ & $65 \%(n=13)$ & $57 \%(n=88)$ \\
\hline Age & $28(19)$ & $20(6)$ & $22.5(8)$ & $23(12)$ \\
\hline Years played & $10(13)$ & $9(7)$ & $11.5(7)$ & $10(9)$ \\
\hline Water polo sessions / week & $3(2)$ & $6(3)$ & $7(5)$ & $4(3)$ \\
\hline
\end{tabular}

Table 2: Point prevalence and past history of shoulder and hip/groin pain, across men and women.

\begin{tabular}{|l|l|l|l|l|}
\hline & & Men & Women & Risk Ratio \\
\hline \multirow{3}{*}{ Shoulder } & Prevalence & $39.4 \%(n=26)$ & $37.5 \%(n=33)$ & $0.95(0.63-1.42)$ \\
\cline { 2 - 5 } & Past History & $78.8 \%(n=52)$ & $83.0 \%(n=73)$ & $1.05(0.90-1.23)$ \\
\hline \multirow{2}{*}{ Hip/groin } & Prevalence & $28.8 \%(n=19)$ & $36.4 \%(n=32)$ & $1.26(0.79-2.02)$ \\
\cline { 2 - 5 } & Past History & $53.0 \%(n=35)$ & $65.9 \%(n=58)$ & $1.24(0.95-1.63)$ \\
\hline
\end{tabular}

a risk factor for the presence of hip/groin pain (RR 1.99 [95\%CI 1.27-3.12]). Current hip pain was also a risk factor for current shoulder pain (RR 1.70 [95\%CI 1.23-2.35]).

No association was found between current shoulder pain and age $(\mathrm{r}=0.08, \mathrm{p}=0.35)$, years played $(\mathrm{r}=0.06, \mathrm{p}=0.46)$, or levels of other physical activity ( $\mathrm{r}=-0.10, \mathrm{p}=0.18)$. No association was found between current shoulder pain and water polo sessions per week $(\mathrm{r}=0.08, \mathrm{p}=0.30)$ or hours of water polo training per week $(\mathrm{r}=0.10, \mathrm{p}=0.24)$.

For hip/groin pain there was no association between presence of pain and age $(r=0.02, p=0.80)$, years played $(r=-0.05, p=0.50)$ or levels of other physical activity $(r=0.11$, $\mathrm{p}=0.17$ ). Number of water polo sessions per week showed weak association to presence of hip/groin pain $(r=0.20$ (95\%CI 0.04-0.35), $\mathrm{p}=0.01$ ); however, hours per week was not significantly associated. $(\mathrm{r}=0.13, \mathrm{p}=0.96)$.

\section{DISCUSSION}

This is the first study to investigate self-reported hip/groin and shoulder pain across different playing levels in water polo players. The results of the current study indicate high rates of both self-reported shoulder and hip/groin pain, with significant impact on performance. While no associations were found between demographic variables, playing level or training load with shoulder pain, playing at more elite levels was associated with increased rates of hip/groin pain, and number of sessions per weak was also weakly associated. These results also show water polo as one of the first sports with equal or higher prevalence of hip/groin pain in women compared to men.

Research is increasing regarding the pathoetiology and management of hip/groin pain in athletes and this is the first time the prevalence of pain has been quantified in water polo players. The eggbeater kick may expose the hip joint to high repetitive shearing loads, which could lead to the development of hip joint related symptoms. The strong

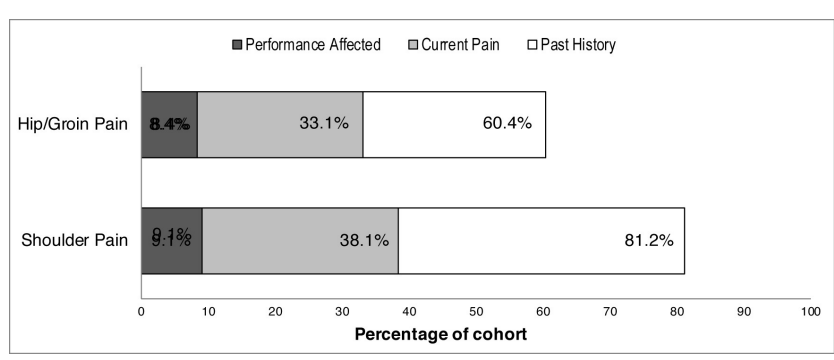

Figure 2: Prevalence and incidence of shoulder and hip/groin pain, broken down by playing level.

Significant risk ratios are presented in brackets.

adduction forces in outer ranges required to elevate the body out of the water while using a breaststroke kick may also predispose these athletes to adductor related groin pain. Additionally, the influence of playing in a non-weight bearing environment may also influence muscle and strength development, which may in turn be a factor in symptom onset. Differences have been described in the gluteal muscles of swimmers compared to land athletes, ${ }^{14}$ but how this could link to development of pain is not yet understood, and may in fact be considered adaptive to environmental factors. Given players at more elite levels had higher prevalence of symptoms, as well as a weak association between number of sessions per week and pain, internal loading may also be a significant factor in this population. Water polo competitions are often in a tournament format, which may expose players to fluctuations in workload. Also training camp environments may result in more eggbeater kick time than in a competition setting, where loads may be more intermittent in a game.

Another explanation for elite players having higher rates of pain may be high amounts of training and play during adolescence. It is reasonable to assume that the reason such players are at the elite level is due to the significant amount 
of time and practice committed to their development at a younger age. While in this study, history of years played was not significantly associated with symptoms, sub-group analysis in different playing levels was not conducted due to small sample size. High loads and lack of movement variability (early sport specialization) may cause alterations in developmental morphology of the hip joint, which may be implicated in symptom development. ${ }^{15}$ This would be similar to research in other sports such as soccer and ice hockey, where higher loads during the adolescence are linked to development of altered hip morphology. ${ }^{16,17}$ However, it is also possible that morphological changes are in fact a normal positive adaptation, ${ }^{18}$ and biomechanical structure is a poor predictor of musculoskeletal pain in a number of body regions. ${ }^{19}$

Shoulder pain continues to be the most common complaint affecting water polo players, and the results of this study support previous anecdotal reporting ${ }^{20}$ and smaller scale studies in water polo players. ${ }^{6,7}$ Combining an overhead throwing sport, with high swimming loads as well as the mechanical disadvantage of being immersed in water places elevated demands on the shoulder complex. Other overhead sports generate significant power from the lower limbs, and while this has not been investigated formally in water polo players, most of the generated energy by the lower limb is used to elevate the body out of the water, and has less influence on forward propulsion of the ball compared to a tennis serve or handball shot. Shoulder pain in these players should not be considered the same as that seen in swimmers, as water polo athletes are likely to have many characteristics of 'throwing shoulders' including adaptive responses such as humeral retrotorsion and muscular and movement imbalances. ${ }^{21}$ Future studies need to compare if and how pain in this population compares to that seen in swimmers, as well as other throwing sports.

No other associations were found between variables measured and rates of pain in this population. Factors not considered in this study include shooting load (number of shots), which result in both high shoulder loads, but also significant force generation at the hip to elevate the body high out of the water using the eggbeater kick. Previous authors have shown number of shots per session to be associated with increased levels of shoulder pain, ${ }^{10}$ however hip and groin symptoms were not evaluated. Information about player position was also not asked in this study. There is likely significant variability between athletes for the number of shots taken per session, which may explain why session duration or frequency did not show any associations with shoulder pain. This would be similar to high variability in jump loads seen between volleyball players. ${ }^{22}$ While the hip and groin complex may also be affected by varying shooting loads, the eggbeater kick is also used for a number of other skills such as ball passing and in defensive situations, and this might explain why sessions per week was only weakly associated with hip/groin symptoms.

An important finding from this survey research is the high presence of concomitant shoulder and hip/groin symptoms. Kinetic chain assessment is often advocated in throwing athletes, and should not be neglected despite a different lower limb "platform" in water polo players. The ability to elevate out of the water or 'jump height' is linked with throwing power, ${ }^{23}$ hence a reduction in lower limb force production may result in associated force changes at the shoulder, which may lead to development of symptoms. It is also possible, that athletes suffering from both were in high workload periods, and not as a result of one injury leading to the other. Emerging research shows the sequential influence of injury in seemingly distant body regions; ${ }^{24}$ however, temporality related to symptoms cannot be determined from this paper. As water polo is predominantly an amateur sport in Australia, unique factors related to the professionalism of the sport may influence findings also, such as lack of time to maximise injury reduction or recovery efforts, as well as improper training techniques.

These novel findings suggest an important area of impact for elite players and clubs to take advantage of to maximize results and performance. Results from this study suggest that at least five to six players per playing roster (of 13-15) could be suffering from pain, with some of this leading to performance deficits. As such, understanding what pathology or patterns of pain are present may allow for programs or treatments to be implemented in order to reduce the burden of pain. While strength and range of motion are often cited as a risk factor for musculoskeletal conditions, ${ }^{7}$ further work is required to confirm this in water polo players. It is plausible that physical capacity must be sufficient to tolerate the high forces around the shoulder and high cumulative movement around the hip. However, given elite players, who could be assumed to be stronger and fitter than their recreational counterparts, seem afflicted more by hip/groin symptoms, other variables may be more important to consider.

There were a number of limitations to this study. The sample size was smaller than had been originally aimed for, and represents only an estimated $2.5 \%$ of the registered members in Australia (WPA). Given this some findings may have been underpowered, or lead to Type I errors. Findings may also be overestimated as a result of injury reporting bias, though care was taken when advertising this study to encourage all members of the community, regardless of injury history to respond. The online survey format may also have resulted in inaccuracies in reporting, especially in sections such as training loads. A decision was made to separate out workload questions regarding water polo, and other activity, to ensure robustness of these responses. For clearer findings regarding the relationship between workload and pain, a more precise method of measuring workload should be used in future. Another possible limitation is the use of the terminology 'hip/groin', which was chosen to capture a general estimate of the problem in this population. This however can also be considered as a strength, as it was up to patients to report their own pain status and use of diagnostic terms or subgroups may have resulted in inaccuracies. Currently there is a lack of research regarding what specific pathologies or diagnoses are responsible for hip/groin pain in water polo players. Further research should investigate whether specific subgroups (such as classification based on the Doha Agreement) ${ }^{2}$ are represented in this population. Finally, side of pain (including whether unilateral or bilateral) and how that related to players' side dominance was not included in the questionnaire, and this may have helped discussing theories around the influence 
of shooting on symptoms.

\section{CONCLUSION}

Australian water polo players across competitive levels reported high rates of shoulder and hip/groin pain. Players at the elite level had higher prevalence and past history of hip/ groin pain. Hip and groin symptoms may present as a result of eggbeater kick loading during skeletal development or as a result of load related factors, however further investigation is required to understand specific pathoeetiology in this population. No associations were found between demographic or training variables and shoulder pain, suggesting more subtle factors such as shooting loads may explain high amounts of pain. Clinicians involved in water polo should assess the entire kinetic chain when investigating any injury, and should ask about concomitant hip/groin issues in athletes presenting with shoulder pain.

\section{FUNDING}

No external financial support

CONFLICTS OF INTEREST

None declared

ETHICAL APPROVAL

The University of Melbourne Human Research Ethics ID: 1648247

Submitted: March 01, 2020 CDT, Accepted: July 24, 2020 CDT 


\section{REFERENCES}

1. Franic M, Ivkovic A, Rudic R. Injuries in water polo. Croat Med J. 2007;48(3):281-288.

2. Weir A, Brukner P, Delahunt E, et al. Doha agreement meeting on terminology and definitions in groin pain in athletes. $\mathrm{Br} J$ Sports Med.

2015;49(12):768-774. doi:10.1136/bjsports-2015-0948 69

3. Spittler J, Keeling J. Water Polo Injuries and Training Methods. Curr Sports Med Rep. 2016;15(6):410-416. doi:10.1249/JSR.0000000000000 $\underline{305}$

4. Mosler AB, Blanch PD, Hiskins BC. The effect of manual therapy on hip joint range of motion, pain and eggbeater kick performance in water polo players. Phys Ther Sport. 2006;7:128-136.

5. Sanders RH. Analysis of the Eggbeater Kick Used to Maintain Height in Water Polo. J Applied Biomech. 1999;15(3):284-291. doi:10.1123/jab.15.3.284

6. Webster MJ, Morris ME, Galna B. Shoulder pain in water polo: a systematic review of the literature. $J$ Sci Med Sport. 2009;12(1):3-11. doi:10.1016/j.jsams.200 7.05.014

7. Miller AH, Evans K, Adams R, Waddington G, Witchalls J. Shoulder injury in water polo: A systematic review of incidence and intrinsic risk factors. J Sci Med Sport. 2018;21(4):368-377. doi:10.10 16/i.jsams.2017.08.015

8. Witwer A, Sauers E. Clinical Measures of Shoulder Mobility in College Water-Polo Players. J Sport Rehab. 2006;15(1):45-57. doi:10.1123/isr.15.1.45

9. McMaster WC, Long SC, Caiozzo VI. Isokinetic torque imbalances in the rotator cuff of the elite water polo player. Am J Sports Med. 1991;19(1):72-75. doi:10.1177/036354659101900112

10. Wheeler K, Kefford T, Mosler A, Lebedew A, Lyons $\mathrm{K}$. The volume of goal shooting during training can predict shoulder soreness in elite female water polo players. J Sci Med Sport. 2013;16(3):255-258. doi:10.1 016/i.jsams.2012.06.006

11. Mountjoy M, Junge A, Benjamen S, et al. Competing with injuries: injuries prior to and during the 15th FINA World Championships 2013 (aquatics). Br J Sports Med. 2015;49(1):37-43. doi:10.1136/bjsport s-2014-093991
12. Bahr R. Why screening tests to predict injury do not work-and probably never will...: a critical review. Br J Sports Med. 2016;50(13):776-780. doi:10.1136/bjs ports-2016-096256

13. Clarsen B, Myklebust G, Bahr R. Development and validation of a new method for the registration of overuse injuries in sports injury epidemiology: the Oslo Sports Trauma Research Centre (OSTRC) overuse injury questionnaire. Br J Sports Med. 2013;47(8):495-502. doi:10.1136/bjsports-2012-09152 $\underline{4}$

14. Semciw AI, Green RA, Pizzari T. Gluteal muscle function and size in swimmers. J Sci Med Sport. 2016;19(6):498-503. doi:10.1016/j.jsams.2015.06.004

15. Feeley BT, Agel J, LaPrade RF. When Is It Too Early for Single Sport Specialization? Am J Sports Med. 2016;44(1):234-241. doi:10.1177/036354651557 $\underline{6899}$

16. Agricola R, Heijboer MP, Ginai AZ, et al. A cam deformity is gradually acquired during skeletal maturation in adolescent and young male soccer players: a prospective study with minimum 2-year follow-up. Am J Sports Med. 2014;42(4):798-806. doi:1 $0.1177 / 0363546514524364$

17. Siebenrock KA, Kaschka I, Frauchiger L, Werlen S, Schwab JM. Prevalence of cam-type deformity and hip pain in elite ice hockey players before and after the end of growth. Am J Sports Med. 2013;41(10):2308-2313. doi:10.1177/03635465134975 $\underline{64}$

18. Silvis ML, Mosher TJ, Smetana BS, et al. High prevalence of pelvic and hip magnetic resonance imaging findings in asymptomatic collegiate and professional hockey players. Am J Sports Med. 2011;39(4):715-721. doi:10.1177/0363546510388931

19. Heerey JJ, Kemp JL, Mosler AB, et al. What is the prevalence of imaging-defined intra-articular hip pathologies in people with and without pain? A systematic review and meta-analysis. Br J Sports Med. 2018;52(9):581-593. doi:10.1136/bjsports-2017-09826 $\underline{4}$

20. Colville JM, Markman BS. Competitive water polo. Upper extremity injuries. Clin Sports Med. 1999;18(2):305-312, vi. 
21. Whiteley R, Oceguera M, Benedict Valencia E, Mitchell T. Adaptations at the Shoulder of the Throwing Athlete and Implications for the Clinician. Techniques in Shoulder and Elbow Surgery.

2012;13(1):36-44. doi:10.1097/BTE.0b013e31823fe84f

22. Bahr MA, Bahr R. Jump frequency may contribute to risk of jumper's knee: a study of interindividual and sex differences in a total of 11,943 jumps video recorded during training and matches in young elite volleyball players. Br J Sports Med.

2014;48(17):1322-1326. doi:10.1136/bjsports-2014-09 $\underline{3593}$
23. McCluskey L, Lynskey S, Leung CK, Woodhouse D, Briffa K, Hopper D. Throwing velocity and jump height in female water polo players: performance predictors. J Sci Med Sport. 2010;13(2):236-240. doi:1 0.1016/i.jsams.2009.02.008

24. Toohey LA, Drew MK, Cook JL, Finch CF, Gaida JE. Is subsequent lower limb injury associated with previous injury? A systematic review and metaanalysis. Br J Sports Med. 2017;51(23):1670-1678. do i:10.1136/bjsports-2017-097500 